\title{
'Pseudo-Dominant' Inheritance in Friedreich's Ataxia: Clinical and Genetic Study of a Brazilian Family
}

\author{
Adriana Moro, MD, ${ }^{1}$ Alberto R.M. Martinez, MD, ${ }^{2}$ Simone C.V. Karuta, MD, ${ }^{1}$ Renato P. Munhoz, MD, MSc, ${ }^{3}$ Mariana Moscovich, MD,
} Francisco M.B. Germiniani, MD, ${ }^{1}$ Walter O. Arruda, MD, MSc, ${ }^{1}$ Salmo Raskin, MD, PhD, ${ }^{4}$ Hélio A.G. Teive, MD, PhD ${ }^{1, *}$

\begin{abstract}
Friedreich's ataxia (FA) is an autosomal recessive inherited disorder characterized by progressive gait and limb ataxia, hypertrophic cardiomyopathy, limb muscular weakness, and areflexia with positive extensor plantar response. Loss of vibratory and position sense, skeletal abnormalities, and dysarthria are common comorbid features. The wide spectrum of disease may lead to a diagnostic challenge, and in such a scenario, the inheritance pattern might be a clue to diagnosis. A rare and peculiar pattern observed in some families is the pseudodominant pattern that is usually characterized by phenotypic variation, which, in turn, could make it even harder to get to a correct diagnosis. This pattern, although seemingly similar to a true dominant pattern of inheritance, should be kept in mind whenever one is evaluating a family with FA. We evaluated a Brazilian family of Italian ancestry with variation of phenotype among affected siblings.
\end{abstract}

First described in 1863 by Nikolaus Friedreich, Friedreich's ataxia (FA) is the most common form of inherited ataxia in Caucasians, ${ }^{1}$ with an estimated point prevalence of 3 in 100,000 and a carrier rate of 1 in 100 to 1 in $50 .^{2}$ Approximately $96 \%$ are homozygous, derived from an expansion of a GAA triplet repetition in the FXN gene on the $9 \mathrm{q} 13$ chromosome, leading to impaired transcription of frataxin, a mitochondrial protein, whose deficit causes the clinical manifestations of FA. $^{3}$

As a neurodegenerative disease, the relentlessly symptom progression involves several systems, as well as the central and peripheral nervous systems, leading to progressive ataxia, sensory neuronopathy, muscular weakness, and the classical feature of loss of tendon reflexes associated with an extensor plantar reflex (Babinski's sign). Non-neurological compromise includes cardiomyopathy, skeletal abnormalities, such as pes cavus and scoliosis, and diabetes. ${ }^{4}$

The most commonly observed inheritance pattern is autosomal recessive, so that it was even considered by Harding in 1981 as a primary aspect for the diagnosis. ${ }^{5}$ However, the wide spectrum of disease may lead to a diagnostic challenge, and in such a scenario, the inheritance pattern might be a clue to correct diagnosis. A rare and peculiar pattern observed in some families is the pseudodominant pattern, which is characterized by phenotypic variation and therefore could make it even harder to reach a correct diagnosis. ${ }^{1,6}$

\section{Patients and Methods}

We evaluated a Brazilian family of Italian ancestry, in which 5 patients from two generations were affected. The pedigree is shown in Figure 1. Three patients were examined at the Movement Disorders Unit of the Neurology Service of the Hospital de Clínicas, Federal University of Paraná (Curitiba, Brazil). Patient II-4 was evaluated at home by one of the authors.

Patients were assessed using a standardized protocol, including demographic, clinical, and paraclinical (blood tests, neurophysiological evaluation, and neuroimaging) data. Signed informed consents were obtained following a protocol approved by the Institutional Ethics Committee of the Hospital de Clínicas, Federal University of Paraná.

\footnotetext{
${ }^{1}$ Movement Disorders Unit, Neurology Service, Internal Medicine Department, Hospital de Clínicas, Federal University of Paraná, Curitiba, Paraná, Brazil; ${ }^{2}$ Neurology Department, Faculty of Medical Sciences, UNICAMP, Campinas, São Paulo, Brazil; ${ }^{3}$ Department of Medicine, Morton and Gloria Shulman Movement Disorders Centre, Toronto Western Hospital, University of Toronto, Toronto, Ontario, Canada; ${ }^{4}$ Genetika Laboratory, Curitiba, Paraná, Brazil

*Correspondence to: Dr. Hélio A.G. Teive, General Carneiro 1103/102, 80060-150 Curitiba, Paraná, Brazil; E-mail: hagteive@mps.com.br Keywords: pseudo-dominant inheritance, Friedreich's ataxia, phenotype.

Relevant disclosures and conflicts of interest are listed at the end of this article.

Received 11 June 2014; revised 20 August 2014; accepted 21 August 2014.

Published online 30 September 2014 in Wiley InterScience (www.interscience.wiley.com). DOI:10.1002/mdc3.12087
} 


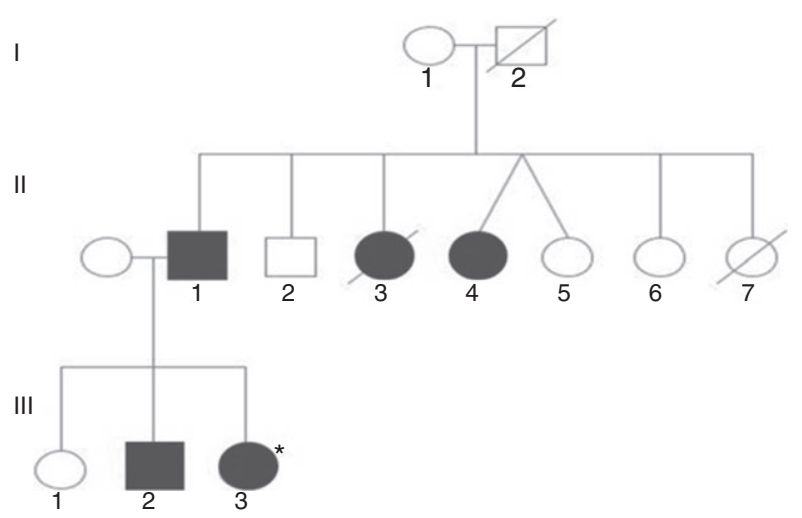

Figure 1 Pedigree of a Brazilian family affected with a pseudodominant pattern of inheritance of FA. Squares indicate males; circles, females. Open symbols indicate unaffected individuals; shaded, affected patients; slashes, deceased individuals; asterisk, index case.

\section{Results}

\section{Case Reports}

\section{Patient II-1}

The patient had a history of progressive ataxia that started when he was 30 years old, which ultimately resulted in his becoming chair bound. On examination, at the age of 68 , he had moderate dysarthria, dysphagia, generalized ataxia, dysmetria, and intentional tremor on finger-to-nose testing and was unable to perform the heel-knee maneuver. Deep tendon reflexes were absent in both upper and lower extremities. He could not sit on the bed unaided, nor was he able to stand or walk, even if supported. Brain MRI showed unspecific microangiophaty, and echocardiogram findings were suggestive of cardiomyopathy. DNA analysis showed two expanded alleles with more than 700 GAA repeats in each one.

His wife has a normal allele and another with more than 900 GAA repeats. Also, there was no consanguinity between the spouses.

\section{Patient II-4}

A 57-year-old female had progressive unsteadiness of gait since she was 18 years old. Later, she would notice difficulty in speaking, and by the age of 37 , she became wheelchair bound as a result of worsening ataxia. She had a history of hypertension, diabetes mellitus, hypothyroidism, and atrial fibrillation. On neurological examination, she had moderate-to-severe dysarthria, bilateral deafness, and square wave jerks. Strength of upper and lower limbs was markedly reduced and she showed distal muscle atrophy. Joint position and vibration sense were diminished; there was loss of tendon reflexes and the plantar cutaneous reflex was indifferent. We also identified cavus feet, lower-limb edema, and altered vascularization (cyanosis of the feet). Electrocardiogram (EKG) examination disclosed an abnor- mal rhythm with atrial fibrillation, without signs of ventricular hypertrophy. She had a twin sister who was asymptomatic.

\section{Patient III-2}

This 39-year-old man began to present with impaired balance when he was 22 years old. Eventually, his ataxia progressed and he lost the ability to walk without assistance. On neurological examination, there was impaired speech, even though it was rather easy to understand it, dysphagia, horizontal nystagmus, and head tremor. Vibrational sensation was diminished and there was loss of tendon reflexes. He exhibited truncal ataxia while sitting and could not stand even with constant support. There was thoracic scoliosis and echocardiographic evidence of cardiomyopathy. There were two expanded alleles on DNA analysis, one with more than 700 GAA repeats and the other containing 900 repeats.

\section{Patient III-3 (Index Case)}

This patient first exhibited clumsiness in handwriting at the age of 26, followed by progressive unsteadiness of gait. On examination, at age 33, she had slurred speech, horizontal nystagmus, unsteady gait, postural tremor of the hands, bilateral loss of the deep-tendon knee reflex, and facial myokymia around the eyes, probably the result of anxiety. The results of routine hematological and biochemical investigations were normal. Brain MRI and electrophysiological examination were normal. EKG examination showed signs of disturbance of ventricular repolarization in the inferior wall, and a transthoracic echocardiogram was normal. DNA analysis showed two different expanded alleles, the shorter allele containing 350 trinucleotide repeats and the longer one containing 1,000 trinucleotide repeats.

\section{Discussion}

FA is often described as "the most common form of hereditary ataxia with a typical autosomal recessive pattern of inheritance and onset of symptoms usually before the age of 20 years." Nevertheless, the estimated prevalence is 3 in $100,000,{ }^{2}$ making it a relatively rare disease in the general population. Nevertheless, these patients will eventually be evaluated by a neurologist who might reach a correct diagnosis by combining both clinical features and laboratorial data to establish a correct diagnosis.

Technological advances had made molecular tests available in daily practice, which makes it relatively easy to confirm the FA hypothesis for a young patient who presents with signs of ataxia, absent tendon reflexes, Babinski's sign, and no known similar cases in the family. Although molecular analysis may be an effective tool given that it provides the clinician with a fast diagnosis, the costs of a primary approach leading to widespread use of molecular analysis should follow a rationale.

An autosomal recessive pattern of inheritance and specific clinical features can provide excellent clues to an FA hypothesis. The pedigree described here contrasts with this expected pattern; rather, we found a "pseudo-dominant" inheritance 
pattern, which could be inadequately interpreted as an autosomal-dominant pattern.

Pseudo-dominancy is a well-known pattern of inheritance in FA first described by Harding in her classic 1980s works, and ever since, few other pedigrees have been published. ${ }^{1,6,7}$ We describe the first known Brazilian FA pedigree with this pattern of inheritance.

Another interesting point regarding pseudo-dominant FA is the phenotypical variability among the affected patients. In the same family, the classical phenotype was observed in 3 patients (II-4, III-2, and III-3), whereas the late-onset presentation was observed in only 1 patient (II-1).

The family presented here provided us with the opportunity to investigate the molecular basis of intrafamilial clinical polymorphism associated with a rare pseudo-dominant pattern of inheritance in FA. We highlight the fact that recessive forms of inheritance may present with a phenotype similar to dominant ones. Therefore, one should consider with caution such cases in order to not avoid performing a genetic evaluation for common recessive disorders, regardless of a seemingly dominant pattern.

\section{Author Roles}

(1) Research Project: A. Conception, B. Organization, C. Execution; (2) Statistical Analysis: A. Design, B. Execution, C. Review and Critique; (3) Manuscript: A. Writing of the First Draft, B. Review and Critique.

A.M.: 1A, 1B, 3A, 3B

A.R.M.M.: 1A, 1B, 3A, 3B

S.C.V.K.: 1A, 1B, 3B

R.P.M.: $3 \mathrm{~B}$

M.M.: 2B, 3B
F.M.B.G.: $3 \mathrm{~B}$

W.O.A.: $3 \mathrm{~B}$

S.R.: $2 \mathrm{~B}, 3 \mathrm{~B}$

H.A.G.T.: 1A, 1C, 2C, 3A, 3B

\section{Disclosures}

Funding Sources and Conflicts of Interest: The authors report no sources of funding and no conflicts of interest.

Financial Disclosures for previous 12 months: The authors declare that there are no disclosures to report.

\section{References}

1. Illarioshkin SN, Bagieva GKH, Klyushnikov SA, Ovchinnikov IV, Markova ED, Ivanova-Smolenskaya IA. Different phenotypes of Friedreich's ataxia within one 'pseudo-dominant' genealogy: relationships between trinucleotide (GAA) repeat lengths and clinical features. Eur J Neurol 2000;7:535-540.

2. Koeppen AH. Friedreich's ataxia: pathology, pathogenesis, and molecular genetics. J Neurol Sci 2011;303:1-12.

3. Martelli A, Napierala M, Puccio H. Understanding the genetic and molecular pathogenesis of Friedreich's ataxia through animal and cellular models. Dis Model Mech 2012;5:165-176.

4. Santos R, Lefevre S, Sliwa D, Seguin A, Camadro JM, Lesuisse E Friedreich ataxia: molecular mechanisms, redox considerations and therapeutic opportunities. Antioxid Redox Signal 2010;13:651-690.

5. Harding AE. Friedreich's ataxia: a clinical and genetic study of 90 families with an analysis of early diagnostic criteria and interfamilial clustering of clinical features. Brain 1981;104:589-620.

6. Webb S, Doudney K, Pook M, Chamberlain S, Hutchinson M. A family with pseudodominant Friedreich's ataxia showing marked variation of phenotype between affected siblings. J Neurol Neurosurg Psychiatry 1999;67:217-219.

7. Harding AE, Zilkha KJ. "Pseudo-dominant" inheritance in Friedreich's ataxia. J Med Genet 1981;18:285-287. 